\title{
Growth hormone excess and sternohyoid muscle mechanics in rats
}

\author{
P. Attal*, V. Claes ${ }^{\#}$, S. Bobin*, P. Chanson ${ }^{\top,+}$, P. Kamenicky ${ }^{\top,+}$, \\ P. Zizzari ${ }^{\S}$ and $Y$. Lecarpentier ${ }^{f * * *}$
}

ABSTRACT: In vitro isotonic and isometric mechanical properties of the sternohyoid (SH) muscle, an upper airway dilator muscle, were studied in rats with a growth hormone (GH)-secreting tumour (GH tumour group; $n=10$ ). The effects of muscle fatigue were also studied.

Stress and shortening were measured in muscles contracting from zero load up to isometric load under tetanic conditions. Isometric stress and maximum unloaded shortening velocity were determined and compared with values obtained from control rats $(n=10)$. Crossbridge kinetics and energetics and mechanical efficiency were calculated from Huxley's equations.

Compared with controls, isometric stress, mechanical efficiency, crossbridge number and crossbridge single force were lower in the GH tumour group. The probability of crossbridge being in the power stroke configuration was lower in the GH tumour group than in controls. Muscle fatigue significantly impaired maximal muscle efficiency and crossbridge single force in the $\mathrm{GH}$ tumour group but not in controls.

In conclusion, mechanical and energetic properties of the $\mathrm{SH}$ muscle and crossbridge properties were worse in the GH tumour group than in controls. This may partly account for impairment of the upper airway dilator muscle function and the increased occurrence of obstructive sleep apnoea in acromegaly.

KEYWORDS: Fatigue, growth hormone, mechanics, myosin, sleep apnoea syndrome, sternohyoid muscle

$\mathrm{U}$ pper airway dilator muscles exhibit phasic activity during inspiration [1, 2]. In synergy with the diaphragm, these muscles contribute to upper airway patency during breathing in awake and sleeping subjects [3]. The negative pressure generated in the upper airways by diaphragm contraction is one of the most important factors involved in the stimulation of the pharyngeal dilator muscles, which promote opening of the upper airways during inspiration [3-5]. The activity of pharyngeal muscles has also been studied in sleep apnoea syndrome, in which repeated occlusion of the upper airways occurs during sleep. Pharyngeal occlusions seem to be associated with a relative decline in activity of upper airway dilator muscles [3,6]. The presence of enlarged pharyngeal muscles associated with weakness in these muscles is a well recognised clinical observation in patients with acromegaly [7]. Administration of growth hormone (GH) to animals produces an increase in muscle bulk [8]. However, during GH hypersecretion syndrome, the contractile function of hypertrophied muscle has been shown to be decreased [9-11]. Changes in mechanical properties of pharyngeal muscles may, thus, have important pathophysiological implications in the development of sleep apnoea syndrome, which has been shown to be prevalent in acromegaly [12, 13].

The aim of the present study was to determine the isotonic and isometric mechanical properties of the sternohyoid (SH) muscle, a pharyngeal dilator muscle, in rats with a GH-secreting tumour. As diaphragm contractility has previously been shown to be impaired in a similar model of rats with a GH-secreting tumour [11], we hypothesised that mechanical performance of the SH muscle may also be decreased. In striated muscle, muscle performance is determined both by the number and the single force of crossbridges, which represent molecular motors generating force and shortening. Using the equations of HUXLEY [14], we determined crossbridge single force, number and kinetics in the $\mathrm{SH}$ muscle of rats with a $\mathrm{GH}$-secreting tumour.

\section{MATERIALS AND METHODS}

\section{Animal model of rats with a $\mathrm{GH}$-secreting} tumour

The investigation adhered to the American Physiological Society's Guiding Principles in the
AFFILIATIONS

*Services de d'Oto-rhinolaryngologie

'Services de d'Endocrinologie, ${ }^{f}$ Services de Physiologie, Hôpital de Bicêtre, Assistance PubliqueHôpitaux de Paris, Université Paris Sud, Le Kremlin-Bicêtre, Paris, +Institut National de la Santé et de la Recherche Médicale, U693 Hôpital de Bicêtre, Paris,

${ }^{\S}$ Institut National de la Santé et de la Recherche Médicale, U894, Université Paris V Descartes, Paris, ${ }^{* \star}$ Centre de Recherche Clinique, Centre Hospitalier Régional de Meaux, Meaux, France, and "Dept of Pharmaceutical Sciences, University of Antwerpen, Wilrijk, Belgium.

\section{CORRESPONDENCE}

Y. Lecarpentier

Service de Physiologie, Hôpital de Bicêtre

Assistance Publique-Hôpitaux de Paris 94275 le Kremlin-Bicêtre France E-mail: yves.lecarpentier@ bct.aphp.fr

Received:

Nov 122008

Accepted after revision: April 012009 First published online: April 082009 
Care and Use of Animals and to the National Institute of Health Guide for Care and Use of Laboratory Animals, and was allowed by the Animal Ethics Committee of the French Institut National de la Santé et de la Recherche Médicale. All efforts were made to minimise pain and suffering and to reduce the number of animals used. GH-hypersecreting cells were cultured in Ham's F10 medium supplemented with 15\% horse serum and $2.5 \%$ fetal calf serum as previously described [15-17]. Briefly, $12 \times 10^{6}$ cells were injected subcutaneously into the flank of 8-week-old female Wistar-Furth rats (Charles River, L'Arbresle, France). Animals were housed in plastic containers, placed in a sound-proof room with controlled temperature $\left(22-24^{\circ} \mathrm{C}\right)$ and illumination (12 h light, $12 \mathrm{~h}$ dark schedule with lights off at 19:00 h), fed ad libitum and weighed weekly. SH muscle mechanical function was studied in isolated muscle strips from rats with a $\mathrm{GH}$-secreting tumour 12 weeks after injection of GH-hypersecreting cells (GH tumour group; $n=10)$. $\mathrm{SH}$ muscle strips were also studied from control animals of the same age $(n=10)$.

\section{Hormonal immunoassays}

After brief anaesthesia, blood samples were immediately collected into heparinised, chilled tubes, and the plasma was stored at $-20^{\circ} \mathrm{C}$ until assay. Plasma GH concentrations were evaluated by enzyme immunoassay (reference preparation (RP)2) as previously described [18]. Values are reported in terms of rat GH RP2 with sensitivity of $0.6 \mathrm{ng} \cdot \mathrm{mL}^{-1}$. The intraand inter-assay coefficients of variation were 4 and $14 \%$ respectively.

\section{Mounting procedure}

Animals were anaesthetised with pentobarbital sodium (60 $\mathrm{mg} \cdot \mathrm{kg}^{-1}$ i.p.). SH muscle strips were removed via a median cervicotomy from the mandible to the manubrium and after separation of the submaxillary glands. The suprahyoidian muscular attachments were cut, leaving the hyoid bone attached to the infrahyoid muscles. The hyoid bone was cut medially and then the two SH muscles were separated along the middle line as far as the inferior end of the muscles, requiring a sternotomy. A strip of $\mathrm{SH}$ muscle (2 $\mathrm{mm}$ width) was obtained by cutting one of the two SH muscles laterally. At the upper end of the strip, a piece of hyoid bone was left (hyoid end) and at the lower end a piece of sternum (sternum end). Each muscle strip was vertically suspended in a bath containing Krebs-Henseleit solution with the following composition: $118 \mathrm{mM} \mathrm{NaCl}, 4.7 \mathrm{mM} \mathrm{KCl}, 1.2 \mathrm{mM} \mathrm{MgSO}_{4} .7 \mathrm{H}_{2} \mathrm{O}$, $1.1 \mathrm{mM} \mathrm{KH} \mathrm{PO}_{4}, 24 \mathrm{mM} \mathrm{NaHCO}, 2.5 \mathrm{mM} \mathrm{CaCl} 2.6 \mathrm{H}_{2} \mathrm{O}$ and $4.5 \mathrm{mM}$ glucose. The solution was bubbled with $95 \% \mathrm{O}_{2} / 5 \%$ $\mathrm{CO}_{2}$ and maintained at $26^{\circ} \mathrm{C}(\mathrm{pH}$ 7.4). The sternum end of the $\mathrm{SH}$ muscle strip was held in a stationary clip at the bottom of the bath, and the hyoid end was held in a spring clip attached to an electromagnetic lever system. Muscles were stimulated by means of two platinum electrodes under tetanic conditions as follows: electrical stimulus $1 \mathrm{~ms}$; stimulation frequency $100 \mathrm{~Hz}$; train duration $200 \mathrm{~ms}$; train frequency 5 trains $\cdot \mathrm{min}^{-1}$. At $26^{\circ} \mathrm{C}$, the $100 \mathrm{~Hz}$ stimulation frequency yielded the maximum tetanic isometric active tension for the $\mathrm{SH}$ pharyngeal muscle. Experiments were carried out at the initial resting length (L0) corresponding to the apex of the active tension versus initial length curve. At the end of the study, the crosssectional area (in $\mathrm{mm}^{2}$ ) was calculated from the ratio of fresh muscle weight to muscle length at L0, assuming a muscle density of $1.06 \mathrm{~g} \cdot \mathrm{mL}^{-1}$.

\section{Electromagnetic lever system}

The lever system was used to perform several tasks: to impose a well determined preload and afterload on the muscle, to measure force exerted on the muscle and to measure length changes in the muscle [19]. Force generation was based on the electromagnetic principle: a lever $30 \mathrm{~mm}$ long was attached to a coil carrying a constant current. The coil was arranged in a constant magnetic field and a well determined torque developed. Hence, a precision current source determined the force at the tip of the lever. This force could be set by decade switches in calibrated steps of $100,10,1$ and $0.1 \mathrm{mN}$, up to a total of $140 \mathrm{mN}$. The error of the measured force was $<0.1 \%$. The equivalent moving mass of the whole system was $155 \mathrm{mg}$ and its compliance was $0.25 \mu \mathrm{m} \cdot \mathrm{mN}^{-1}$.

The displacement of the lever was measured by means of an opto-electronic transducer. A small diaphragm on the lever modulated the light from a tiny infrared light-emitting diode falling on a photodiode with a daylight filter that blocked visible light but allowed near-infrared radiation to pass. The photodiode current was converted to a voltage and filtered with an active third-order low-pass filter (cut-off frequency $150 \mathrm{~Hz}$ ) with optimal step response. The resulting length range was $5 \mathrm{~mm}$ at a full-scale output voltage of $10 \mathrm{~V}$ (error 1\%) with a noise floor $<2.5 \mu \mathrm{m}$ peak-to-peak.

Force carried by the muscle preparation was measured with unilateral feedback techniques: the displacement signal was continuously compared with a reference voltage, corresponding to the stop position of the lever and, hence, the resting length of the muscle. The servo loop kept the lever at this stop position as long as the muscle developed a force smaller than the total imposed load. However, if the muscle was stimulated and developed more force than this total load, the feedback became inactive, resulting in the muscle carrying this load during its isotonic contraction-relaxation cycle. The lever behaved as if a mechanical stop was guarding it against stretching the muscle beyond its resting length. The force signal filter had the same characteristics as aforementioned, eliminating a time skew between the force and displacement signal. Maximum range was $140 \mathrm{mN}\left(20 \mathrm{mN} \cdot \mathrm{V}^{-1}\right)$, with a noise floor $<0.05 \mathrm{mN}$.

All analyses were based on digital recordings obtained from a data acquisition system connected to a computer. Two signals, force and length, were recorded. Although data could be loaded in an Excel worksheet, a custom-made program was written to determine the most common muscle parameters automatically. It also calculated the first derivative of both channels to obtain the velocity of shortening and the rate of change of force.

\section{Mechanical analysis, crossbridge kinetics and energetics}

The mechanical parameters of the muscle strips were calculated from two contractions at L0 and recorded in tetanus mode. The first contraction was abruptly clamped to zero-load $3 \mathrm{~ms}$ after the onset of the electrical stimulus. The second contraction was fully isometric. The following mechanical indices were used: maximum unloaded shortening velocity of 
contraction 1 (Vmax) and total force, which was the peak isometric force of contraction 2. Velocity was expressed in $\mathrm{L} 0 \cdot \mathrm{s}^{-1}$, and force was normalised per muscle cross-sectional area (in $\mathrm{mm}^{2}$ ) of the $\mathrm{SH}$ muscle strip, i.e. stress $(\mathrm{P})$ in $\mathrm{mN} \cdot \mathrm{mm}^{-2}$ and time in $\mathrm{s}$.

The hyperbolic stress-velocity $(\mathrm{P}-\mathrm{V})$ relationship was derived from the peak velocity $(\mathrm{V})$ of seven to 10 isotonic afterloaded contractions, plotted against the isotonic load level normalised per cross-sectional area $(\mathrm{P})$, by successive load increments, from zero-load up to the isometric stress ( $\mathrm{P} 0)$. The $\mathrm{P}-\mathrm{V}$ relationship was fitted according to Hill's equation $(\mathrm{P}+\mathrm{a})$ $(\mathrm{V}+\mathrm{b})=(\mathrm{P} 0+\mathrm{a}) \mathrm{b}$ where $-\mathrm{a}$ and $-\mathrm{b}$ are the asymptotes of the hyperbola [20]. For each SH muscle strip, the P-V relationship was accurately fitted by a hyperbola (each $r>0.98$ ). The $G$ curvature of Hill's hyperbola is equal to $\mathrm{P}_{0} / \mathrm{a}=\mathrm{Vmax} / \mathrm{b}[20,21]$.

The equations of HuXLEY [14] were used to calculate the maximum turnover rate of myosin ATPase under isometric conditions (kcat; in $\mathrm{s}^{-1}$ ), the elementary force per single crossbridge interaction (in $\mathrm{pN}$ ) and the number of active crossbridges per cross-sectional area (for more details please see the Appendix) $[22,23]$. Huxley's equations were also used to calculate crossbridge kinetics (fig. 1), i.e. constants of crossbridge attachment ( $\mathrm{f} 1$; in $\mathrm{s}^{-1}$ ) and detachment (g1 and g2; in $\mathrm{s}^{-1}$ ), mean crossbridge velocity during the power stroke (in $\left.\mu \mathrm{m} \cdot \mathrm{s}^{-1}\right)$, duration of the power stroke ( $t \mathrm{~s}$; in $\left.\mathrm{ms}\right)$, the total duration of the crossbridge cycle under isometric conditions $\left(t_{c}\right.$; in $\left.\mathrm{ms}\right)$, and peak efficiency (Effmax). Probabilities of three steps of the crossbridge cycle, i.e. the detachment step (D1), the

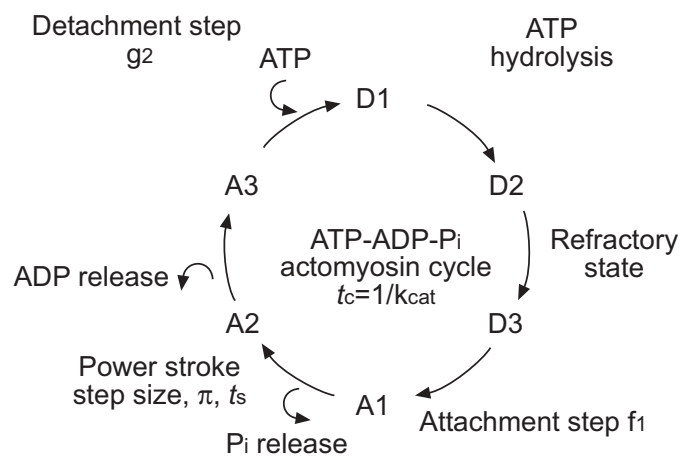

FIGURE 1. The ATP-ADP-Pi-actomyosin crossbridge cycle. The crossbridge cycle was subdivided into six different conformational states, with three detached states (D1, D2 and D3) and three attached states (A1, A2 and A3). The A3 $\rightarrow$ D1 transition was the ATP-binding step; crossbridge detachment occurred when ATP bound to the actin (A)-myosin (M) complex (AM) and the rate constant for detachment was g2; $A M \rightarrow A+M$. The $D 1 \rightarrow D 2$ transition was the ATP hydrolysis; $\mathrm{M}+\mathrm{ATP} \rightarrow \mathrm{M}-\mathrm{ADP}-\mathrm{Pi}$. The $\mathrm{D} 2 \rightarrow \mathrm{D} 3$ transition was $\mathrm{M}-\mathrm{ADP}-\mathrm{Pi} \rightarrow \mathrm{M}^{*}-\mathrm{ADP}-\mathrm{Pi}$, where $\mathrm{M}^{*}$ is myosin in refractory state [24]. The $D 3 \rightarrow A 1$ transition was the attachment state: the myosin head $\left(\mathrm{M}^{*}-\mathrm{ADP}-\mathrm{Pi}\right)$ was weakly bound to $\mathrm{A}$ and the rate constant for attachment was $\mathrm{f} 1$; M-ADP-Pi+A $\rightarrow$ AM-ADP-Pi. The $\mathrm{A} 1 \rightarrow \mathrm{A} 2$ transition was the power stroke, a strongly bound, high-force state which was triggered by Pi release: AM$\mathrm{ADP}-\mathrm{Pi} \rightarrow \mathrm{AM}-\mathrm{ADP}+\mathrm{Pi}$; ts was the duration of the power stroke, step size was the amplitude of the myosin head movement and $\pi$ was the crossbridge unitary force. The $A 2 \rightarrow A 3$ transition was the release of the hydrolysis product ADP: AM$A D P \rightarrow A M+A D P ; t c$ was the duration of the overall crossbridge cycle and $k c a t=1 / t c$, where kcat was the maximum turnover rate of myosin ATPase under isometric conditions. attachment step (A1) and the power stroke step (A2), were also calculated (fig. 1 and the Appendix).

\section{Fatigue protocol}

Muscle fatigue was induced by repeatedly stimulating each strip by means of 75 trains $\cdot \mathrm{min}^{-1}$ of $200 \mathrm{~ms}$ duration at a frequency of $100 \mathrm{~Hz}$ under isometric conditions. Total force was monitored and was found to decrease progressively. Stimulation continued until the muscle was fatigued to the point where it generated $\sim 50 \%$ of baseline total force. Then mechanical parameters were determined in tetanic mode using similar methods to those used before fatigue.

\section{Statistical analysis}

Data are expressed as mean \pm SEM. Controls were compared with the GH tumour group using an unpaired t-test after ANOVA; p-values $<0.05$ were required to rule out the null hypothesis. Linear regression was based on the least squares method. The asymptotes - $\mathrm{a}$ and -b of Hill's hyperbola were calculated by linear regression and the least squares method.

\section{RESULTS}

\section{Effects of tumour growth on body weight and plasma hormone levels}

At the time of sacrifice, the mean body weight of the $\mathrm{GH}$ tumour group was significantly higher than that of the controls. In the $\mathrm{GH}$ tumour group, the mean \pm SEM weight of the tumour was $13.2 \pm 1.8 \mathrm{~g}$ at the time of the study, i.e. 12 weeks after injection of GH-hypersecreting cells. Plasma levels of $\mathrm{GH}$ were markedly higher in $\mathrm{GH}$ tumour group rats than in control rats (table 1). The length of SH muscle did not differ significantly between the two groups.

\section{Mechanics}

P0, total number of active crossbridges per $\mathrm{mm}^{2}$ and the single force of crossbridges were significantly lower in the $\mathrm{GH}$ tumour group than in controls (fig. 2). Vmax did not differ between the two groups (fig. 2b). The economy of contraction

\begin{tabular}{|c|c|c|c|}
\hline \multirow[t]{2}{*}{ TABLE 1} & \multirow[b]{2}{*}{ Control group } & \multirow[b]{2}{*}{ GH tumour group } & \multirow[b]{2}{*}{ p-value } \\
\hline & & & \\
\hline Body weight g & $207 \pm 3.7$ & $437.4 \pm 16.4$ & $<0.001$ \\
\hline Muscle strip length mm & $31.2 \pm 2.3$ & $31.3 \pm 5.3$ & NS \\
\hline GH plasma levels $\mu \mathrm{g} \cdot \mathrm{L}^{-1}$ & $8.1 \pm 4.3$ & $6807 \pm 3523$ & $<0.001$ \\
\hline a $\mathrm{mN} \cdot \mathrm{mm}^{-2}$ & $17.56 \pm 6.8$ & $10.72 \pm 4.18$ & $<0.05$ \\
\hline b L $0 . \mathrm{s}^{-1}$ & $1.09 \pm 0.37$ & $1.52 \pm 0.43$ & $<0.05$ \\
\hline G & $4.42 \pm 1.39$ & $3.23 \pm 0.92$ & $<0.05$ \\
\hline ab $\mathrm{mN} \cdot \mathrm{mm}^{-2} \cdot \mathrm{L}_{0} \cdot \mathrm{s}^{-1}$ & $19.9 \pm 12.6$ & $16.4 \pm 8.1$ & NS \\
\hline$f_{1} s^{-1}$ & $326 \pm 110$ & $456 \pm 128$ & $<0.05$ \\
\hline$g 1 s^{-1}$ & $87 \pm 55$ & $159 \pm 80$ & $<0.05$ \\
\hline g2 $\mathrm{s}^{-1}$ & $1828 \pm 394$ & $1820 \pm 409$ & NS \\
\hline \multicolumn{4}{|c|}{$\begin{array}{l}\text { Data are presented as mean } \pm \text { SEM, unless otherwise stated. -a and }-\mathrm{b} \text { : } \\
\text { asymptotes of the Hill hyperbola; G: curvature of the hyperbola; } a b \text { : } \\
\text { maintenance heat; } \mathrm{f} 1 \text { : crossbridge attachment constant; g1 and g2: crossbridge } \\
\text { detachment constants; NS: nonsignificant. }\end{array}$} \\
\hline
\end{tabular}


was worse in the $\mathrm{GH}$ tumour group than in controls as reflected by the significant decrease in Effmax and G curvature (table 1 and fig. $3 b$ ). The asymptote $-\mathrm{a}$ of the $\mathrm{P}-\mathrm{V}$ hyperbola was significantly lower in the GH tumour group than in controls (table 1), although the contrary was observed for the asymptote $-b$. The maintenance heat under isometric conditions did not differ between the two groups (table 1).

\section{Crossbridge kinetics and energetics}

Constants of attachment (f1) and detachment (g1) were significantly higher in the GH tumour group than in controls (table 1). However, g2, the detachment constant after the step size, did not differ between the two groups (table 1). The kcat was also significantly higher in the GH tumour group (fig. 3a). Both the $t_{s}$ and the $t_{c}$ were significantly shorter in the $\mathrm{GH}$ tumour group than in controls (fig. $3 \mathrm{c}$ and d). The probabilities of steps D1 and A1 were significantly higher in the GH tumour group than in controls (fig. $4 \mathrm{a}$ and $\mathrm{b}$ ). The probability of step A2 did not differ between the groups (fig. 4c). Mean crossbridge velocity during the stroke was significantly higher in the GH tumour group than in controls (fig. 4d).

\section{Effects of fatigue}

In both groups, muscles were fatigued to a similar extent $(\sim 50 \%$ of $\mathrm{P} 0$; table 2$)$. Vmax was significantly reduced in the
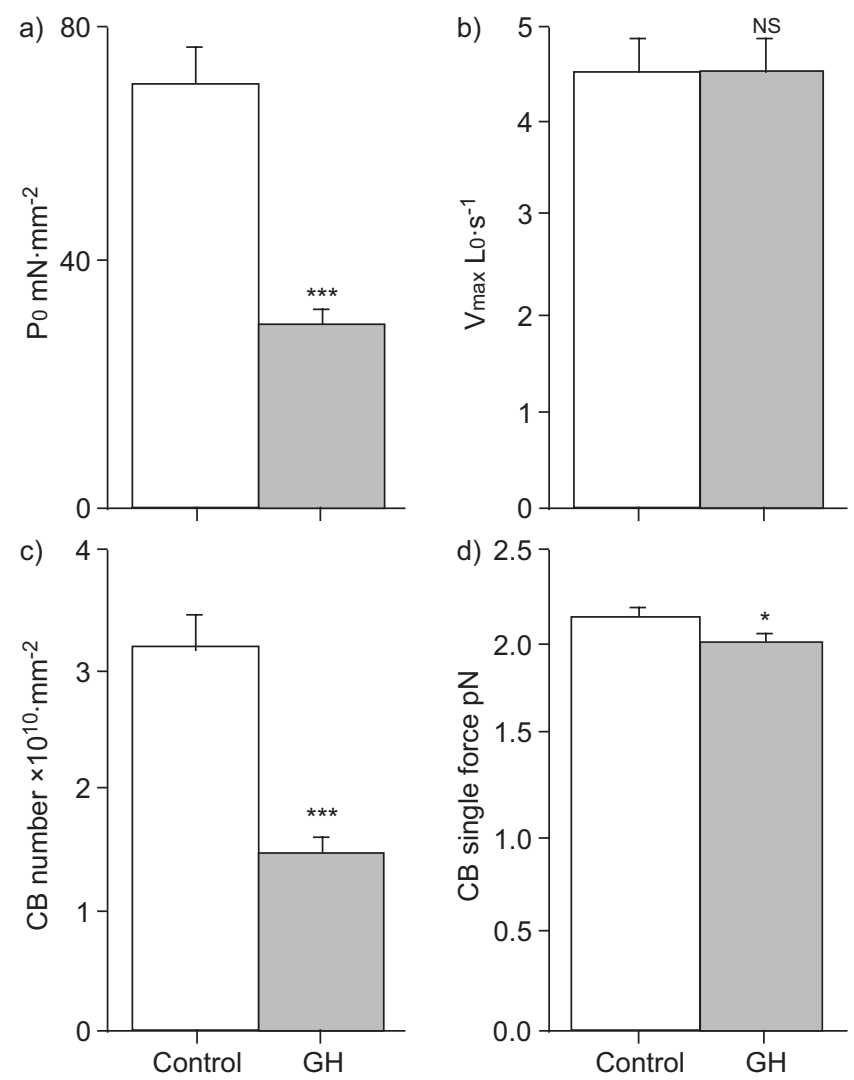

FIGURE 2. a) Isometric stress (P0); b) maximum unloaded shortening velocity (Vmax); c) number of active crossbridges (CBs) per cross-sectional area; d) the elementary force per single CB. Data are presented as mean \pm SEM for controls ( $\square$ ) and the growth hormone $(\mathrm{GH})$ tumour group $(\square)$. NS: nonsignificant; *: $p<0.05$; $\star * *: p<0.001$. controls $(p<0.05)$ whereas the decrease of $V \max$ in the $\mathrm{GH}$ tumour group was nonsignificant. Fatigue impaired the mechanics and energetics of both the GH tumour group and the controls. Indeed, fatigue impaired the myothermal economy of contraction more in the GH tumour group than in controls, as indicated by the significant decrease in G curvature of the $\mathrm{P}-\mathrm{V}$ relationship, Effmax and crossbridge single force (table 2).

\section{Relationships between parameters}

In the study groups as a whole, there was a direct linear relationship between the $\mathrm{P} 0$ and the number of active crossbridges per cross-sectional area (fig. 5a). A linear relationship was also found between kcat and crossbridge velocity during the stroke (fig. 5c). No relationship existed between $\mathrm{P} 0$ and the elementary force per single crossbridge (fig. 5b). A curvilinear relationship was demonstrated between the single crossbridge force and the G curvature (fig. 5d).

\section{DISCUSSION}

We demonstrated that there were intrinsic alterations in the $\mathrm{SH}$ muscle mechanical performance in rats with a $\mathrm{GH}$-secreting tumour. The fall in total tension was attributed to the decline in both the number and unitary force of crossbridge myosin molecular motors. Moreover, muscle fatigue significantly impaired crossbridge single force and muscle efficiency in the GH tumour group, but not in controls (table 2). This may be important, as upper airway dilator muscles have a respiratory function. They participate in preventing upper airway collapse when intrapharyngeal pressure becomes negative during inspiration. The pharynx is the only part of the large airways that lacks rigid support, since its anterior wall is surrounded only by soft tissue [25]. During sleep or unconsciousness, striated muscle tone is decreased, which reduces the muscular support of the pharyngeal area [26]. This contributes to passive prolapse of the tongue and the soft palate and, consequently, favours obstruction of airflow [27]. This mechanism is aggravated if pharyngeal mechanical performance is impaired [28]. Failure of upper airway dilator muscles to maintain pharyngeal patency during sleep plays a role in the development of obstructive sleep apnoea. The ability to generate stress in vitro may differ from the ability to generate force in vivo and, therefore, the ability to create outer wall stress on the upper airways may not be changed. However, sleep apnoea syndrome is known to occur in clinical conditions involving pharyngeal neuromuscular dysfunction [28]. Thus, a high prevalence of sleep apnoea has been reported in Charcot-Marie-Tooth disease and the severity of the sleep apnoea syndrome has been found to correlate with the severity of changes in motor nerve conduction. This suggests that neurogenic upper airway myopathy may contribute to sleep apnoea syndrome [29]. Moreover, oculopharyngeal muscular dystrophy, which selectively affects the extra-ocular and pharyngeal muscles, has been reported to be associated with sleep apnoea syndrome in the absence of obesity or abnormal upper airway morphology [30]. Thus, a myopathy can be associated with upper airway collapse that occurs uniquely during sleep and results in sleep apnoea syndrome. These studies show that upper airway myopathies may induce contractile dysfunction and sleep apnoea syndrome. 

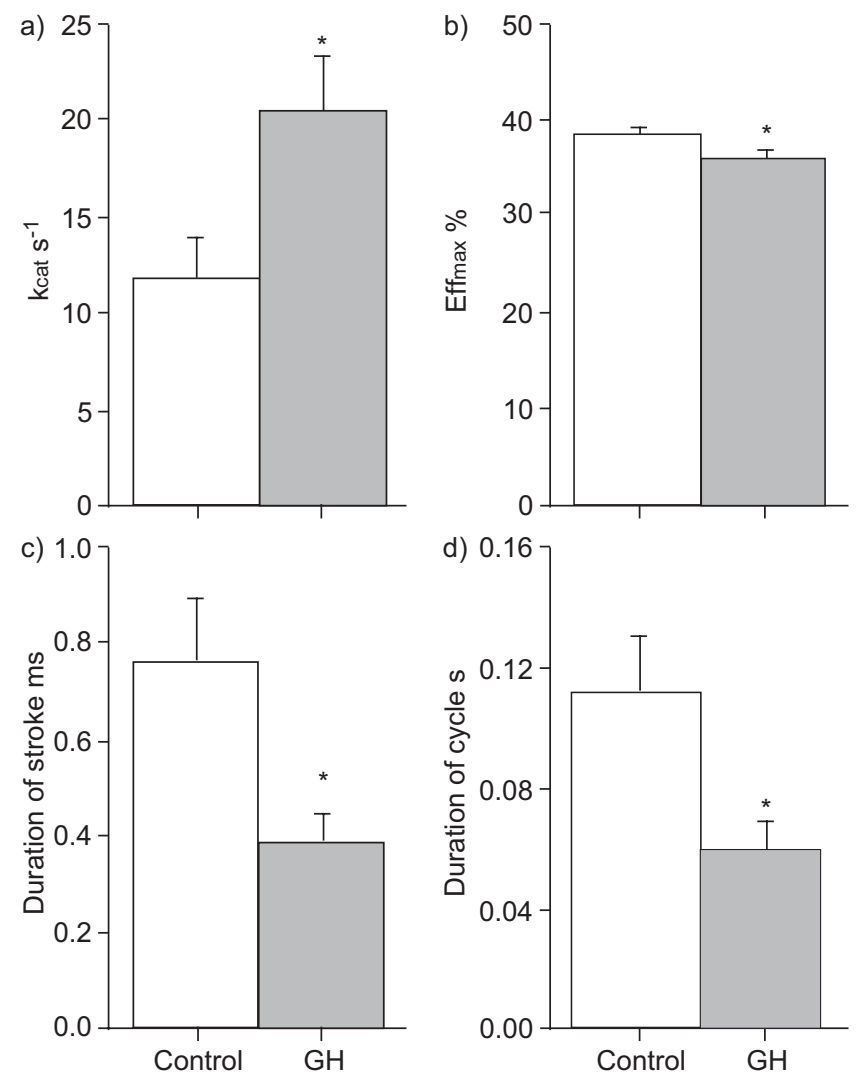

FIGURE 3. a) Maximum turnover rate of myosin ATPase under isometric conditions (kcat); b) peak mechanical efficiency (Effmax); c) duration of the power stroke step; d) total duration of the crossbridge cycle under isometric conditions. Data are presented as mean \pm SEM for controls $(\square)$ and the growth hormone $(\mathrm{GH})$ tumour group ( $\square)$. *: $p<0.05$.

\section{Myopathy in GH hypersecretion syndrome}

GREENBAUM and Young [8] found a general hypertrophy in various muscles in rats treated with pituitary extracts. BIGLAND and JeHRING [9] showed that tetanic isometric tension of hypertrophied quadriceps was decreased in rats treated with GH. In GH-secreting Wistar-Furth rats, it has been found that the size of type I muscle fibres was increased to the greatest extent, whereas type IIA fibres were unaffected [31].

Histological and histochemical studies have been performed on muscle biopsies in acromegalic patients. MASTAGLIA [32] has described several abnormalities, namely isolated fibre necrosis or vacuolar degeneration, increased numbers of internal nuclei, high glycogen content, and either hypertrophy of type I and type II fibres or atrophy of both fibre types; and using electron microscopy also found large amounts of lipofuscin pigment in many fibres, while some fibres had large sarcolemmal nuclei with prominent nucleoli and a prominent Golgi apparatus. NAGULESPAREN et al. [7] have described hypertrophy of type I muscle fibres and atrophy of type II fibres. In other studies performed on acromegalic patients, histopathological analyses of skeletal muscle have shown sclerotic lesions, hypertrophic fibres, fibre degeneration, calcifications, altered mitochondria (elongation, matrical pallor, and cristae abnormalities), infiltration of glycogen granules, inclusion bodies, excess lipofuscin pigment and myofilament
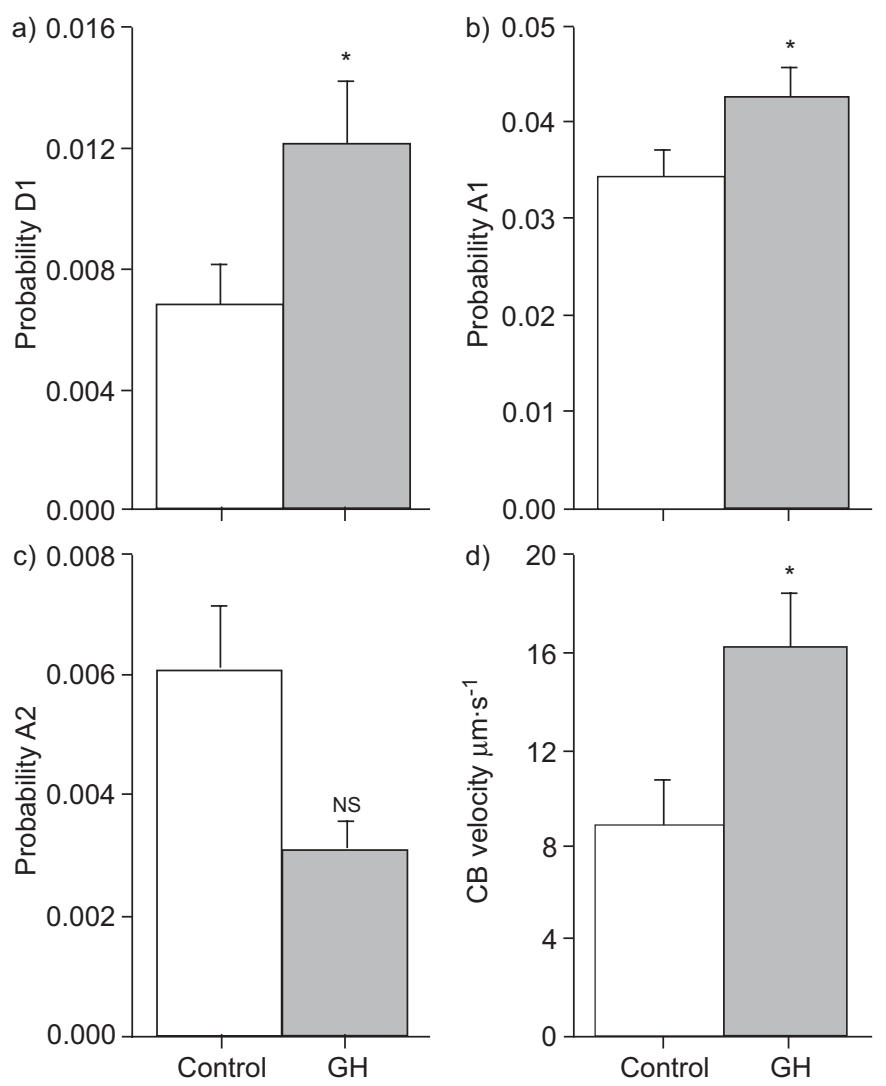

FIGURE 4. Probabilities of three steps of the crossbridge (CB) cycle and $\mathrm{CB}$ velocity during the stroke. a) Probability of the detachment step (D1); b) probability of the attachment step (A1); c) probability of the power stroke step (A2); d) CB velocity during the stroke. Data are presented as mean \pm SEM for controls $(\square)$ and the growth hormone $(\mathrm{GH})$ tumour group $(\square)$. NS: nonsignificant; *: $p<0.05$.

loss, and vesicular dilatations [33-35]. Histological abnormalities can partly explain muscle weakness in patients with $\mathrm{GH}$ hypersecretion. This cellular damage may also partly explain the decrease in crossbridge density per cross-sectional area observed in our study (fig. 2c).

Upper airway dilator muscles have been shown to be faster and less resistant to fatigue than the diaphragm $[36,37]$. In the rat, the myosin fibre type composition of the $\mathrm{SH}$ muscle is $5 \%$ type I, $28 \%$ type IIa and $67 \%$ type IIb. That of the diaphragm is $42 \%$ type I, $31 \%$ type IIa and $27 \%$ type Ilb [38]. The diaphragm, with a high type I fibre percentage, appears to be better adapted than the $\mathrm{SH}$ muscle to continuously overcoming elastic and resistive loads during breathing.

\section{Crossbridge modifications in rats with a GH-secreting tumour}

The precise cellular and molecular effects of the GH on striated muscles of the GH-secreting tumour rat model used in the present study appear to be complex and dependent on the type of sarcomeric muscle. Thus, total isometric tension and the G curvature of the $\mathrm{P}-\mathrm{V}$ relationship have been found to be increased in heart muscle [16], contrary to observations in diaphragm [11] and SH muscles (table 1 and fig. 2). Where crossbridges are concerned, changes in the kinetics of attachment and detachment (table 1 and fig. 1) induced an increase 


\begin{tabular}{|c|c|c|}
\hline \multirow[t]{2}{*}{ TABLE 2} & \multicolumn{2}{|c|}{$\begin{array}{l}\text { Effects of fatigue on sternohyoid muscle in } \\
\text { control and growth hormone }(\mathrm{GH}) \text { tumour } \\
\text { groups }\end{array}$} \\
\hline & Control group & GH group \\
\hline Po & $-54 \pm 5^{\star}$ & $-46 \pm 9^{*}$ \\
\hline$V_{\max }$ & $-21 \pm 3^{*}$ & $-7 \pm 2$ \\
\hline G & $-18 \pm 9$ & $-32 \pm 7^{*}$ \\
\hline Effmax & $-7 \pm 4$ & $-16 \pm 2^{*}$ \\
\hline$\pi$ & $-6 \pm 4$ & $-13 \pm 2^{*}$ \\
\hline \multicolumn{3}{|c|}{$\begin{array}{l}\text { Data are presented as mean } \pm \text { SEM of percentage changes induced by fatigue } \\
\text { versus baseline. Po: total isometric stress; Vmax: maximum unloaded shortening } \\
\text { velocity; G: curvature of Hill's hyperbola; Effmax: peak mechanical efficiency; } \pi \text { : } \\
\text { crossbridge single force. *: } \mathrm{p}<0.05 \text { for fatique versus baseline. }\end{array}$} \\
\hline
\end{tabular}

in probabilities of steps A1 and D1 in the GH tumour group (fig. 4). Conversely, the probability of step A2, the power stroke step of the crossbridge cycle, did not change. Together with the decrease in crossbridge number and crossbridge single force, this partly explained the decrease in contractile performance in the GH tumour group (fig. 2). In diaphragm muscle of the same animal model, only the crossbridge number has been found to be decreased, the crossbridge single force remaining similar to controls [11]. This shows that deleterious effects of massive GH hypersecretion on crossbridge mechanics and energetics were more marked in $\mathrm{SH}$ muscle than in diaphragm [39]. This is corroborated by the decreased muscle efficiency observed in the $\mathrm{SH}$ muscle but not in the diaphragm [39]. Differences in the fibre type composition between the two muscles may partly explain these results. Both the crossbridge velocity and kcat were increased in the GH tumour group compared with controls, but remained linearly related (fig. 5). Moreover, fatigue in rat $\mathrm{SH}$ muscle has been found to induce unfavourable mechanical behaviour [39]. However, in the present study, the Vmax was not modified in the GH tumour group (fig. 2), due to the absence of change in the detachment constant g2 (table 1) [14]. Similar behaviour has been previously observed in heart muscle in the same $\mathrm{GH}$ rat model [16]. The decrease in $\mathrm{G}$ curvature of the $\mathrm{P}-\mathrm{V}$ relationship paralleled the decrease in crossbridge single force according to a curvilinear relationship inherent to Huxley's equations (table 1 and fig. 5) [14, 23].

\section{Effects of fatigue}

It has been previously shown that effects of fatigue for a given species depend on the muscle concerned [39] and for a given muscle depend on the studied species. In rat diaphragm muscle, fatigue induces an increase in both the $G$ curvature and muscle efficiency, although the contrary was observed in rat $\mathrm{SH}$ muscle. Differences in fibre type composition may partly explain these observations, with $\sim 90 \%$ of fast type II fibres in rat $\mathrm{SH}$ and with a mixed fibre muscle composition in rat diaphragm muscle $[37,38,40]$. In the present study, fatigue impaired the economy of muscle contraction and crossbridge single force in the GH tumour group, although the effect of fatigue was not significant in controls (table 2). Fatigue exacerbated the decrease in $\mathrm{SH}$ muscle efficiency and crossbridge
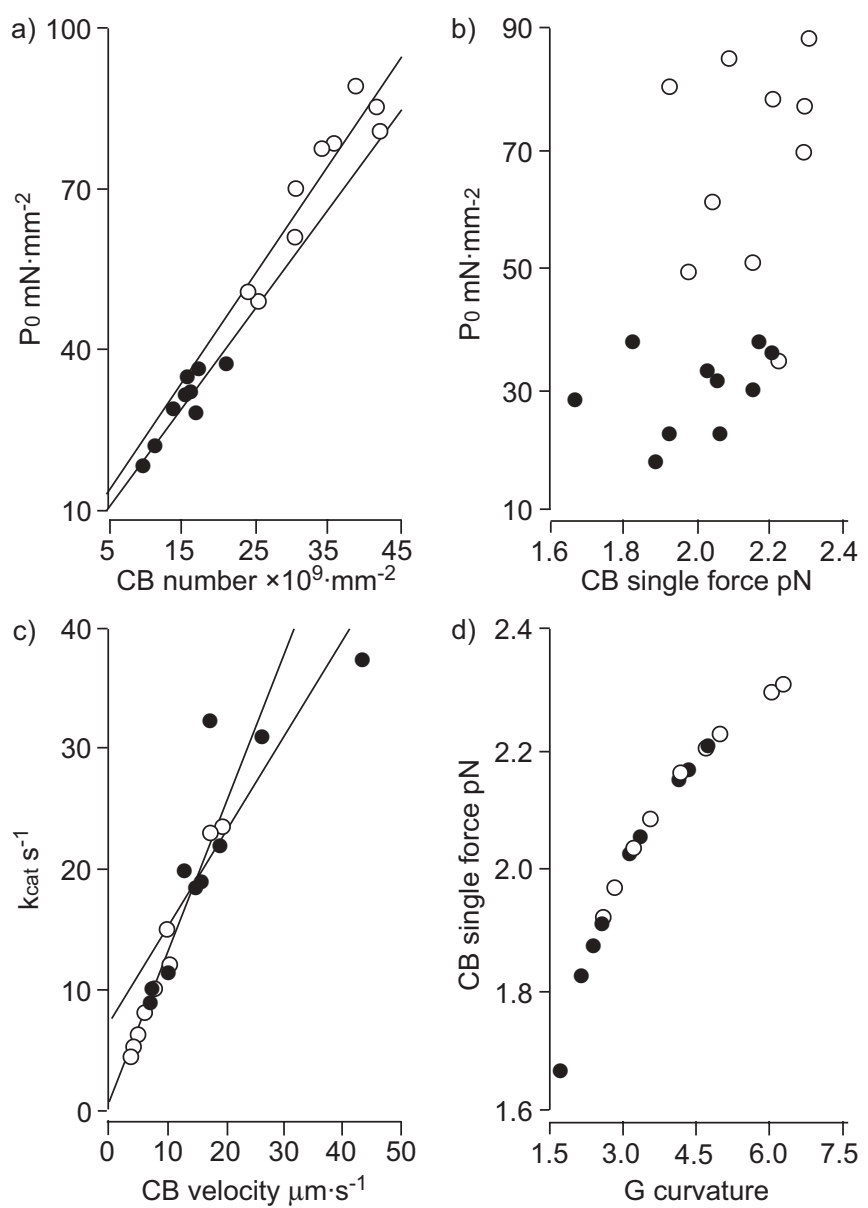

FIGURE 5. a) Linear relationship between isometric stress (Po) and active crossbridge (CB) number per cross-sectional area in controls $(O ; r=0.970)$ and the growth hormone $(\mathrm{GH})$ tumour group $(\bullet ; r=0.904)$; b) relationship between $\mathrm{P} 0$ and the elementary force per single $\mathrm{CB}$; c) linear relationship between the maximum turnover rate of myosin ATPase under isometric conditions (kcat) and CB velocity during the stroke in controls $(r=0.977)$ and the $\mathrm{GH}$ tumour group $(r=0.853)$; $d)$ curvilinear relationship between elementary force per single $\mathrm{CB}$ and the $\mathrm{G}$ curvature.

single force, already observed at baseline in the GH tumour group compared with baseline controls. Excessive fatigue and sensitivity to fatigue of the SH muscle may lead to increased pharyngeal resistance, inducing a vicious circle with aggravation of fatigue and failure of pharyngeal dilator muscle function.

\section{Limitations}

The results pertain strictly to the animal species and experimental conditions used. Mechanics and energetics of myosin crossbridges in human $\mathrm{SH}$ muscle probably differ from those of rat $\mathrm{SH}$ muscle. Moreover, the levels of plasma $\mathrm{GH}$ were dramatically higher in rats with a GH-secreting tumour than those observed in human acromegaly.

\section{Clinical relevance}

It is important to consider the function of the pharyngeal dilator muscles during GH hypersecretion in humans. Sleep apnoea syndrome is prevalent in acromegaly [13] and has been 
shown to increase morbidity and mortality [12]. Indeed, death due to respiratory disease has been found to be three times more likely in acromegaly [41]. Several respiratory disorders have been previously described in patients with GH hypersecretion syndrome [42]. Pneumomegaly has been attributed to an increase in the size or number of alveoli. GH hypersecretion increases upper airway resistance and this has been thought to aggravate respiratory disorders due to an increase in the work of breathing, which may lead to diaphragm fatigue and/or weakness. In humans, narrowing of the upper airways with the development of airflow obstruction has many aetiological factors in acromegaly: hypertrophy of soft tissues of the tongue, pharynx and larynx, structural modification of facial bones and, notably, a development and rotation of the mandibula with retroposition of the tongue. An increase in upper airway resistance induces an increase in the work of the pharyngeal dilators in order to open the airways during inspiration. In association with intrinsic alterations of muscular cells due to GH hypersecretion, this may partly account for the impairment of pharyngeal and diaphragm strength [1], which promotes obstructive sleep apnoea. Finally, GH hypersecretion results in generalised weakness and wasting of skeletal muscles.

\section{Conclusion}

$\mathrm{GH}$ hypersecretion in rats induced major intrinsic impairment of the mechanical performance of the $\mathrm{SH}$ muscle, one of the pharyngeal dilator muscles. The mechanical and energetic profile of the $\mathrm{SH}$ muscle was characterised by a significant fall in both crossbridge number and crossbridge single force, decreased muscle efficiency and alterations in crossbridge kinetics.

\section{APPENDIX}

\section{Crossbridge characteristics}

The rate of total energy release $\left(\mathrm{E}_{\mathrm{Hux}}\right)$ and the isotonic tension $\left(\mathrm{P}_{\text {Hux }}\right)$ as a function of muscle velocity $(\mathrm{V})$ are given by the equations of HUXLEY [14]:

$$
\mathrm{E}_{\text {Hux }}=\mathrm{Ne} \frac{\mathrm{h}}{2 \mathrm{l}} \frac{\mathrm{f}_{1}}{\mathrm{f}_{1}+\mathrm{g}_{1}}\left\{\mathrm{~g}_{1}+\mathrm{f}_{1} \frac{\mathrm{V}}{\phi}\left(1-\mathrm{e}^{-\frac{\phi}{\nabla}}\right)\right\}
$$

$\mathrm{P}_{\text {Hux }}=\mathrm{N} \frac{\mathrm{sW}}{2 \mathrm{l}} \cdot \frac{\mathrm{f}_{1}}{\mathrm{f}_{1}+\mathrm{g}_{1}}\left[1-\frac{\mathrm{V}}{\phi}\left(1-\mathrm{e}^{\frac{\phi}{\mathrm{v}}}\right)\left(1+\frac{1}{2}\left(\frac{\mathrm{f}_{1}+\mathrm{g}_{1}}{\mathrm{~g}_{2}}\right)^{2} \frac{\mathrm{V}}{\phi}\right)\right]$

where $\mathrm{N}$ is the cycling crossbridge number per $\mathrm{mm}^{2}$ at peak isometric tension; $\mathrm{s}$ is the resting optimal sarcomere length $(\mathrm{s}=2.2 \mu \mathrm{m}) ; \mathrm{f} 1$ is the peak value of the rate constant for crossbridge attachment; and g1 and g2 are the peak values of the rate constants for crossbridge detachment. The tilt of the myosin head relative to actin varies from zero to $h$; $f 1$ and g1 correspond to a tilt equal to zero, and g2 corresponds to a tilt $>\mathrm{h} ; \phi=(\mathrm{f} 1+\mathrm{g} 1) \mathrm{h} / \mathrm{s}=\mathrm{b}$.

$\mathrm{w}$ is the maximum mechanical work of a single crossbridge $(\mathrm{w} / \mathrm{e}=0.75)$ [14]. According to the theory of HuXLEY [14], one ATP molecule is split per crossbridge cycle. The standard free energy $\Delta \mathrm{G}^{\circ \prime}$ ATP, e is equal to $5.1 \times 10^{-20} \mathrm{~J}[21] ; \Delta \mathrm{G}^{\circ \prime}$ ATP has been found to be $-32 \mathrm{~kJ} \cdot \mathrm{mol}^{-1}$ at $\mathrm{pH} 7.0$ and $37^{\circ} \mathrm{C}$ [43].

The parameter 1 is the distance between successive actin sites with which any one myosin site can combine. In the work of HUXLEY [14], the calculated estimate of 1 was found to be $15.3 \mathrm{~nm}$. This value corresponds approximately to the meridional spot at $14.3 \mathrm{~nm}$ determined by means of X-ray diffraction and arising from the helical arrangement of crossbridges on the thick filament [44]. This is of the same order of magnitude as the distance corresponding to three successive G-actin monomers. For a given actin filament, the distance 1 between two actin sites with which a myosin site can combine was chosen to be equal to $14.3 \mathrm{~nm}$. The molecular step size $\mathrm{h}$ is defined by the translocation distance of the actin filament per ATP hydrolysis, produced by the swing of the myosin head [45]. Huxley's equations assume that 1 is much greater than $h$. The value of $\mathrm{h}$ was chosen to be equal to $5 \mathrm{~nm}$, a value supported by optical tweezer experiments on muscle myosin II [46].

Calculations of $\mathrm{f} 1, \mathrm{~g} 1$ and $\mathrm{g} 2$ are given by the following equations [23]:

$$
\begin{gathered}
\mathrm{G}=\mathrm{f} 1 / \mathrm{g} 1 \\
\mathrm{~g} 1=\mathrm{swb} / \mathrm{ehG} \\
\mathrm{g} 2=\mathrm{sVmax} / \mathrm{h} \\
\mathrm{kcat}=(\mathrm{h} / 2 \mathrm{l}) \times((\mathrm{f} 1 \mathrm{~g} 1) /(\mathrm{f} 1+\mathrm{g} 1)) \\
\pi=(\mathrm{w} / \mathrm{l}) \times((\mathrm{f} 1) /(\mathrm{f} 1+\mathrm{g} 1))
\end{gathered}
$$

The number of active crossbridges $(\mathrm{N})$ is equal to the ratio of the peak isometric tension and the elementary crossbridge force $(\pi)$. The rate of mechanical work $(\mathrm{WM})$ is given by the equation $\mathrm{WM}=\mathrm{P}_{\mathrm{Hux}} \mathrm{V}$. At any given load level, the mechanical efficiency (Eff) of the muscle is defined as the ratio of WM to $\mathrm{E}_{\mathrm{Hux}}$, i.e. $\mathrm{Eff}=\mathrm{WM} / \mathrm{E}_{\mathrm{Hux}}$, and Effmax is the peak value of Eff.

\section{SUPPORT STATEMENT}

P. Attal was Associate Professor at the University Paris Sud from September 1, 2005 to August 31, 2007.

\section{STATEMENT OF INTEREST}

None declared.

\section{REFERENCES}

1 Sauerland EK, Harper RM. The human tongue during sleep: electromyographic activity of the genioglossus muscle. Exp Neurol 1976; 51: 160-170.

2 Sherrey JH, Pollard MJ, Megirian D. Respiratory functions of the inferior pharyngeal constrictor and sternohyoid muscles during sleep. Exp Neurol 1986; 92: 267-277.

3 Remmers JE, deGroot WJ, Sauerland EK, et al. Pathogenesis of upper airway occlusion during sleep. J Appl Physiol 1978; 44: 931-938.

4 Mathew OP. Upper airway negative-pressure effects on respiratory activity of upper airway muscles. J Appl Physiol 1984; 56: 500-505.

5 Horner RL, Innes JA, Murphy K, et al. Evidence for reflex upper airway dilator muscle activation by sudden negative airway pressure in man. J Physiol 1991; 436: 15-29.

6 Fogel RB, Malhotra A, White DP. Sleep. 2: pathophysiology of obstructive sleep apnoea/hypopnoea syndrome. Thorax 2004; 59: 159-163. 
7 Nagulesparen M, Trickey R, Davies MJ, et al. Muscle changes in acromegaly. Br Med J 1976; 2: 914-915.

8 Greenbaum AL, Young FG. Distribution of protein in the tissues of rats treated with anterior-pituitary growth-hormone. Nature 1950; 165: 521-522.

9 Bigland B, Jehring B. Muscle performance in rats, normal and treated with growth hormone. J Physiol 1952; 116: 129-136.

10 Wolf E, Wanke R, Schenck E, et al. Effects of growth hormone overproduction on grip strength of transgenic mice. Eur J Endocrinol 1995; 133: 735-740.

11 Lecarpentier Y, Coirault C, Riou B, et al. Diaphragm strength and cross-bridge properties during chronic growth hormone hypersecretion. Eur Respir J 1999; 13: 1070-1077.

12 Colao A, Ferone D, Marzullo P, et al. Systemic complications of acromegaly: epidemiology, pathogenesis, and management. Endocr Rev 2004; 25: 102-152.

13 Davi' MV, Dalle Carbonare L, Giustina A, et al. Sleep apnoea syndrome is highly prevalent in acromegaly and only partially reversible after biochemical control of the disease. Eur J Endocrinol 2008; 159: 533-540.

14 Huxley AF. Muscle structure and theories of contraction. Prog Biophys Biophys Chem 1957; 7: 255-318.

15 Bertherat J, Timsit J, Bluet-Pajot MT, et al. Chronic growth hormone $(\mathrm{GH})$ hypersecretion induces reciprocal and reversible changes in mRNA levels from hypothalamic GH-releasing hormone and somatostatin neurons in the rat. J Clin Invest 1993; 91: 1783-1791.

16 Timsit J, Riou B, Bertherat J, et al. Effects of chronic growth hormone hypersecretion on intrinsic contractility, energetics, isomyosin pattern, and myosin adenosine triphosphatase activity of rat left ventricle. J Clin Invest 1990; 86: 507-515.

17 Kamenicky P, Viengchareun S, Blanchard A, et al. Epithelial sodium channel is a key mediator of growth hormone-induced sodium retention in acromegaly. Endocrinology 2008; 149: 3294-3305.

18 Ezan E, Laplante E, Bluet-Pajot MT, et al. An enzyme immunoassay for rat growth hormone: validation and application to the determination of plasma levels and in vitro release. J Immunoassay 1997; 18: 335-356.

19 Sys SU, De Keulenaer GW, Brutsaert DL. Physiopharmacological evaluation of myocardial performance: how to study modulation by cardiac endothelium and related humoral factors? Cardiovasc Res 1998; 39: 136-147.

20 Hill AV. The heat of shortening and the dynamic constants of muscle. Proc R Soc Lond B Biol Sci 1938; 126: 136-195.

21 Woledge RC, Curtin AN, Homsher E. Energetic Aspects of Muscle Contraction. London, Academic Press, 1985.

22 Coirault C, Lambert F, Joseph T, et al. Developmental changes in crossbridge properties and myosin isoforms in hamster diaphragm. Am J Respir Crit Care Med 1997; 156: 959-967.

23 Lecarpentier Y, Chemla D, Blanc FX, et al. Mechanics, energetics, and crossbridge kinetics of rabbit diaphragm during congestive heart failure. FASEB J 1998; 12: 981-989.

24 Eisenberg E, Hill TL, Chen Y. Cross-bridge model of muscle contraction. Quantitative analysis. Biophys J 1980; 29: 195-227.

25 Eckert DJ, Malhotra A. Pathophysiology of adult obstructive sleep apnea. Proc Am Thorac Soc 2008; 5: 144-153.
26 Worsnop C, Kay A, Pierce R, et al. Activity of respiratory pump and upper airway muscles during sleep onset. J Appl Physiol 1998; 85: 908-920.

27 Wheatley JR, Tangel DJ, Mezzanotte WS, et al. Influence of sleep on response to negative airway pressure of tensor palatini muscle and retropalatal airway. J Appl Physiol 1993; 75: 2117-2124.

28 Kimoff RJ. Upperairway myopathy is important in the pathophysiology of obstructive sleep apnea. J Clin Sleep Med 2007; 3: 567-569.

29 Dematteis M, Pépin JL, Jeanmart M, et al. Charcot-Marie-Tooth disease and sleep apnoea syndrome: a family study. Lancet 2001 357: 267-272.

30 Dedrick DL, Brown LK. Obstructive sleep apnea syndrome complicating oculopharyngeal muscular dystrophy. Chest 2004; 125: 334-336.

31 Prysor-Jones RA, Jenkins JS. Effect of excessive secretion of growth hormone on tissues of the rat, with particular reference to the heart and skeletal muscle. J Endocrinol 1980; 85: 75-82.

32 Mastaglia FL. Pathological changes in skeletal muscle in acromegaly. Acta Neuropathol 1973; 24: 273-286.

33 Cheah JS, Chua SP, Ho CL. Ultrastructure of the skeletal muscles in acromegaly - before and after hypophysectomy. Am J Med Sci 1975; 269: 183-187.

34 Pickett JB, Layzer RB, Levin SR, et al. Neuromuscular complications of acromegaly. Neurology 1975; 25: 638-645.

35 Khaleeli AA, Levy RD, Edwards RH, et al. The neuromuscular features of acromegaly: a clinical and pathological study. J Neurol Neurosurg Psychiatry 1984; 47: 1009-1015.

36 van Lunteren E, Salomone RJ, Manubay $\mathrm{P}$, et al. Contractile and endurance properties of geniohyoid and diaphragm muscles. J Appl Physiol 1990; 69: 1992-1997.

37 Dick TE, van Lunteren E. Fiber subtype distribution of pharyngeal dilator muscles and diaphragm in the cat. J Appl Physiol 1990; 68: 2237-2240.

38 Bracher A, Coleman R, Schnall R, et al. Histochemical properties of upper airway muscles: comparison of dilator and nondilator muscles. Eur Respir J 1997; 10: 990-993.

39 Attal P, Coirault C, Chemla D, et al. Isotonic mechanics of a pharyngeal dilator muscle and diaphragm in the rat before and after fatigue. Eur Respir J 2000; 15: 308-313.

40 Petrof BJ, Kelly AM, Rubinstein NA, et al. Effect of hypothyroidism on myosin heavy chain expression in rat pharyngeal dilator muscles. J Appl Physiol 1992; 73: 179-187.

41 Hochban W, Ehlenz K, Conradt R, et al. Obstructive sleep apnoea in acromegaly: the role of craniofacial changes. Eur Respir J 1999; 14: 196-202.

42 Trotman-Dickenson B, Weetman AP, Hughes JM. Upper airflow obstruction and pulmonary function in acromegaly: relationship to disease activity. Q J Med 1991; 79: 527-538.

43 Veech RL, Lawson JW, Cornell NW, et al. Cytosolic phosphorylation potential. J Biol Chem 1979; 254: 6538-6547.

44 Squire JM. The Structural Basis of Muscular Contraction. New York, Plenum Press, 1981.

45 Huxley HE. The mechanism of muscular contraction. Science 1969; 164: 1356-1365.

46 Molloy JE, Burns JE, Kendrick-Jones J, et al. Movement and force produced by a single myosin head. Nature 1995; 378: 209-212. 